\title{
Simulação e estudos de caso no ensino de planejamento e controle da produção: um survey com professores da engenharia de produção
}

\author{
Leonel José Girottia*, Marco Aurélio de Mesquita ${ }^{a}$ \\ a"Universidade de São Paulo, São Paulo, SP, Brasil, leo_girotti@hotmail.com
}

\begin{abstract}
Resumo
A geração de valor depende das prioridades competitivas (custo, qualidade, flexibilidade, serviço e entrega) que apoiam a estratégia empresarial. 0 papel dos Sistemas de Planejamento e Controle da Produção é fornecer informação e modelos para gestão eficiente dos recursos produtivos. Esta pesquisa trata do ensino dos tópicos MRP e APS/PF (programação finita) nos cursos de PCP. Os objetivos incluem levantar as práticas de ensino desses tópicos, focando nas estratégias simulação e estudos de caso, e avaliar se os professores diferenciam as duas abordagens de programação. 0 método empregado foi um survey com professores da Engenharia de Produção. Os resultados indicam que ambos os tópicos são amplamente abordados nos cursos (MRP: 100\% abordam e 91\% o consideram importante; APS: 81\% e $72 \%$, respectivamente). Destaca-se também que 78\% consideram importante diferenciar as abordagens e que $85 \%$ acreditam que o uso de estudos de caso com software contribui para a melhoria do aprendizado.
\end{abstract}

Palavras-chave

Ensino. Planejamento e Controle da Produção (PCP). Survey.

\section{Introdução}

Ao longos dos últimos 30 anos, os sistemas MRP evoluíram gradualmente, transformando-se em sistemas de planejamento hieráriquico que integram necessidades de materiais e capacidade. Mais recentemente, os sistemas Enterprise Resources Planning (ERP) e Advanced Planning and Scheduling (APS) ampliaram as potencialidades de integração e otimização do planejamento de materiais e da capacidade (Hvolby \& Steger-Jensen, 2010).

Segundo Jacobs \& Weston Junior (2007), os sistemas baseados na lógica Material Requirements Planning (MRP) tornaram-se padrão, primeiro para a função da administração de materiais, evoluindo anos depois para sistemas mais completos de Planejamento e Controle da Produção (PCP), denominados Manufacturing Resources Planning (MRPII).

0 modelo MRP determina a quantidade e o momento em que se necessita dos módulos, componentes e matérias-primas para produzir uma determinada quantidade de produtos finais, conforme

especificado no programa mestre de produção, considerando os níveis de estoque e os prazos de suprimento (Orlicky, 1975).

Paralelamente à difusão dos sistemas MRP, os sistemas de programação finita foram sendo aprimorados. Com os avanços na Tecnologia da Informação (Tl) e aumento da complexidade dos processos industriais, esses sistemas, inicialmente chamados de FCS (Finite Capacity Scheduling), foram conquistando espaço (Ovacik, 2011).

Atualmente, essas ferramentas integram os sistemas APS, que disponibilizam modelos de apoio à decisão para os diferentes níveis de planejamento da operação, incluindo a programação detalhada da produção (Stadtler, 2005).

Uma das maiores diferenças entre as abordagens MRP e APS/PF a serem compreendidas refere-se à lógica de carga (entendida como operações que devem ser executadas nos recursos produtivos). Convencionou-se chamar carga finita, quando se 
considera que a carga em um período de tempo deve ser limitada à capacidade efetiva dos recursos (lógica seguida nos APS/PF). Já a lógica de carga infinita representa o conceito de carga ilimitada dos recursos (lógica do MRP).

Os avanços nos conceitos de Planejamento e Controle da Produção (como, por exemplo, o desenvolvimento dos sistemas APS) permitem refletir sobre o processo de ensino-aprendizagem, motivando a busca de melhorias no modo de ensinar essas questões. A utilização de simuladores ou ferramentas computacionais empregadas em ambientes reais de trabalho (por exemplo, softwares comerciais de Programação da Produção) pode ajudar os professores em sua missão e proporcionar aos alunos uma experiência de aprendizagem mais significativa. 0 uso de estudos de caso também poderia ser considerado como uma alternativa interessante para aprimorar o processo de ensino-aprendizagem.

Portanto, reforça-se a necessidade de um melhor entendimento dessas duas abordagens de programação (MRP e APS/PF). Este trabalho tem como objetivo verificar como esses conceitos são abordados nos cursos de graduação em Engenharia de Produção e verificar se o uso da simulação e de estudos de caso pode aprimorar a aprendizagem dos alunos.

Para alcançar os objetivos da pesquisa, emprega-se o método de levantamento por amostragem ou survey, utilizando-se de um questionário autoaplicado. 0 público alvo são os professores que ministram a disciplina Planejamento e Controle da Produção nos cursos de Engenharia de Produção em universidades públicas e privadas.

0 presente artigo encontra-se estruturado da seguinte forma: a seção 1 formula o problema e objetivos da pesquisa, na seção 2 apresenta-se uma revisão de conceitos de programação detalhada da produção, com destaque para as abordagens MRP e APS/PF; a seção 3 discute características das estratégias de ensino por simulação e estudos de caso; na seção 4 detalham-se a metodologia de pesquisa e o questionário; a seção 5 apresenta os resultados e a seção 6 finaliza o artigo com as conclusões.

\section{Programação detalhada da produção}

\subsection{Estratégias de produção}

Lustosa et al. (2008) apresentam três estratégias básicas de produção intermitente:

- Produção para estoque (Make-to-Stock - MTS);

- Produção contra pedido (Make-to-Order - MTO);

- Montagem contra pedido (Assemble-to-Order - AT0).

A estratégia MTS envolve a produção em lotes de produtos padronizados, utilizando ordens de produção que devem ser programadas na produção. Nesse sistema, o fornecedor fabrica os produtos e os vende a partir do estoque de produtos acabados. 0 lead time (entendido como o tempo entre a entrada de um pedido e a sua entrega) é o menor (vide Figura 1). 0 cliente tem pouco envolvimento na especificação dos produtos. Um dos desafios nessa
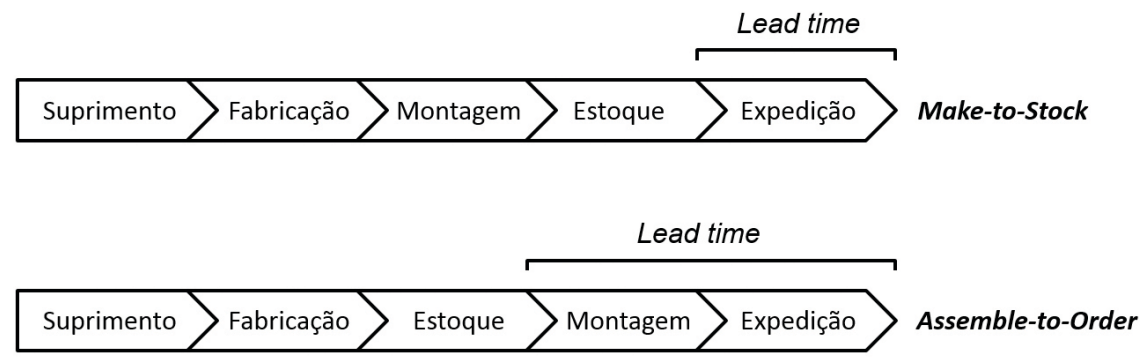

Lead time

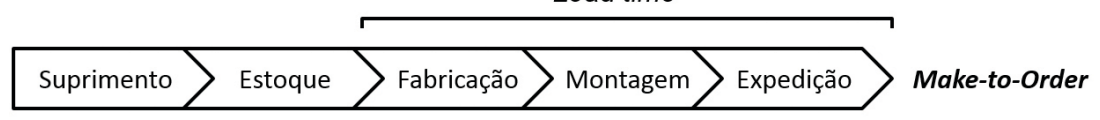

Lead time

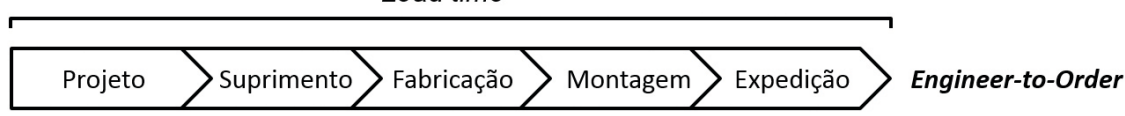

Figura 1. Lead times para cada estratégia de produção. Fonte: adaptado de Arnold (2011). 
estratégia é obter previsões de demanda acuradas, já que as decisões de produção (Programa Mestre de Produção) são baseadas nelas. Busca-se balancear o nível de serviço com os custos de estoques de produto acabado (Arnold, 2011).

A estratégia MTO caracteriza-se por volumes de produção menores, produtos com roteiros diferentes e maior flexibilidade (equipamentos multifuncionais e operadores qualificados). As decisões de produção baseiam-se em pedidos firmes. 0 produto final é usualmente feito de itens padrões, podendo incluir também componentes customizados. 0 lead time é maior do que no MTS, pois inclui as fases de fabricação e montagem. 0 estoque é mantido na entrada do processo (matérias-primas e componentes). Conforme Dumond (2005), os dois maiores desafios que essa estratégia impõe são: a fixação de datas de entrega viáveis e uma programação eficiente dos pedidos em carteira.

A terceira estratégia (ATO) caracteriza-se como um híbrido das anteriores. 0 produto é montado desde componentes padrões (semiacabados) estocados. A montagem do produto final inicia-se com o pedido do cliente. 0 lead time inclui a montagem e a expedição, sendo um valor intermédio entre os valores correspondentes em MTS e MT0 (Arnold, 2011).

Uma quarta estratégia de produção é a denominada Engineering-to-Order (ETO). Semelhante ao MTO, porém no ETO as especificações do cliente requerem um desenho de engenharia diferenciado. Os materiais normalmente são comprados apenas após a definição do projeto e emissão da ordem de produção. 0 lead time é mais longo, por incluir todas as fases do pedido, do projeto à produção (Arnold, 2011).

Embora o problema de programação detalhada da produção esteja presente em todos os ambientes de produção, eles são mais prementes nos ambientes de produção contra pedido (MTO e ETO). Nesses casos, as ferramentas de programação finita apresentam potencial ainda maior de aplicação (Stevenson et al., 2005).

Um aspecto relevante para o desempenho da programação detalhada refere-se ao perfil da carteira de pedidos. Quando a carteira apresenta uma carga elevada de trabalho (muitas ordens com prazo curto) fica mais difícil programar. Vale lembrar que os prazos das ordens em um ambiente MTO são previamente negociados com os clientes. É importante que os responsáveis por esse processo tenham informação e critérios para negociar prazos com os clientes, considerando tanto a viabilidade de atendimento do pedido em questão como também o impacto dele nas ordens já programadas. Slotnick (2011) apresenta uma detalhada revisão desse processo, designado pela sigla OAS de Order Acceptance and Scheduling.

\subsection{Sistemas $M R P$}

Os Sistemas MRP empregam o conceito de demanda dependente, que permite determinar as necessidades de matérias-primas e componentes a partir da demanda independente (Davis et al., 2001). Os dados de entrada básicos do MRP são:

- Programa mestre de produção (MPS - Master Production Schedule): Especifica as quantidades e datas de entrega dos produtos com demanda independente;

- Lista de materiais (BoM - Bill of Material): É um cadastro que, para cada produto, descreve os componentes necessários para sua elaboração;

- Controle de estoques: Fornece a posição de estoque de cada item;

- Cadastro dos itens: Apresenta dados dos itens (código, descrição, fornecedores, unidades de medida, prazo de entrega ou lead time etc).

Hopp \& Spearman (2008) resumem o algoritmo MRP nos seguintes passos:

- Netting (balanço de estoque): Calcular as necessidades líquidas em cada período, subtraindo das necessidades brutas os estoques projetados e os recebimentos programados;

- Lot sizing (formação de lote): Agrupar as necessidades líquidas em lotes de produção ou compra;

- Time phasing (programação para trás): Determinar as datas de liberação das ordens subtraindo das datas de entrega o lead time correspondente;

- BoM explosion (explosão de materiais): Gerar as necessidades brutas de cada componente do próximo nível da lista de materiais;

- Iteração: Respeitando a hierarquia dos itens nas listas de materiais, repetir o processo para os demais componentes.

0 horizonte de planejamento é divido em períodos, denominados janelas de tempo (time buckets), usualmente dias ou semanas. 0 resultado principal da rodada do MRP são as ordens de compra e produção por período (Davis et al., 2001).

Hopp \& Spearman (2008) explicam que o MRP é um sistema tipicamente empurrado, dado que a liberação das ordens é feita com base em uma programação. Sem um sistema eficaz de controle, corre-se o risco de aumento de filas e atrasos decorrentes de incertezas dos processos de suprimento e produção.

Para contornar os efeitos da hipótese de carga ilimitada do MRP, que pode gerar programas de 
produção inviáveis, foram incorporados ao sistema dois módulos de verificação da capacidade, aplicados antes e depois da rodada do MRP. A segunda geração do MRP foi designada Manufacturing Resources Planning ou simplesmente MRP 11 (Wight, 1984).

Conforme Jacobs \& Weston Junior (2007), a evolução continuada dos sistemas MRP 11 resultou nos atuais sistemas integrados de gestão ERP (Enterprise Resources Planning), onde a lógica MRP continua presente nos módulos de Planejamento da Produção.

\subsection{Sistemas APS}

Como mostra a Figura 2, os sistemas APS não se limitam ao nível operacional. Apoiados no crescente poder computacional, esses sistemas foram agregando novos módulos nos diferentes níveis de decisão (curto, médio e longo prazo) e funções (suprimento, produção, distribuição e vendas) do planejamento. Neste artigo, utiliza-se a sigla APS/PF para destacar o módulo de Programação Finita da Produção do APS.

Destacado em cinza está o módulo foco deste trabalho. Como se observa, encontra-se posicionado no horizonte de curto prazo e referenciado à etapa de produção.

Os dados necessários para rodar o módulo de programação detalhada, de acordo com Plenert \& Kirchmier (2000), são:

- Cadastro dos centros: Com código de identificação, descrição e informação acerca da capacidade disponível (turnos, quantidade de máquinas, rendimentos etc.);
- Roteiros: Definem a sequência de operações e tempos (setup e operação) dos processos de produção de cada produto;

- Arquivo de ordens: Tabela das ordens abertas e planejadas conforme a programação. Cada registro inclui basicamente código de identificação, produto, prazo, quantidade e prioridade.

Além dessas informações, deve-se considerar um calendário com os dias e turnos disponíveis para produção, que podem variar entre centros e setores da fábrica. Narasimhan et al. (1995) denominam-no calendário de planejamento da fábrica (The Shop Planning Calendar).

Com as ordens definidas, inicia-se a programação, que consiste em determinar as datas de início e fim de cada operação, respeitando-se as precedências no roteiro e a disponibilidade dos recursos. Esses sistemas, em teoria, produzem programas viáveis, pois consideram a priori as restrições de capacidade. Devido às incertezas dos processos e da demanda, os programas raramente serão cumpridos exatamente, impondo a necessidade de controle e eventual reprogramação. Geralmente, a programação é visualizada em tabelas e nos clássicos gráficos de Gantt (Herrmann, 2006).

Harrison \& Petty (2002) destacam os seguintes desafios da implantação de sistemas de programação finita: i) são sistemas mais complexos, ii) requerem elevada quantidade de dados, iii) dificuldade de controle dos custos e prazos dos projetos de implantação, iv) exigem uma nova qualificação dos programadores, e v) requerem a manutenção constante da base de dados técnicos.

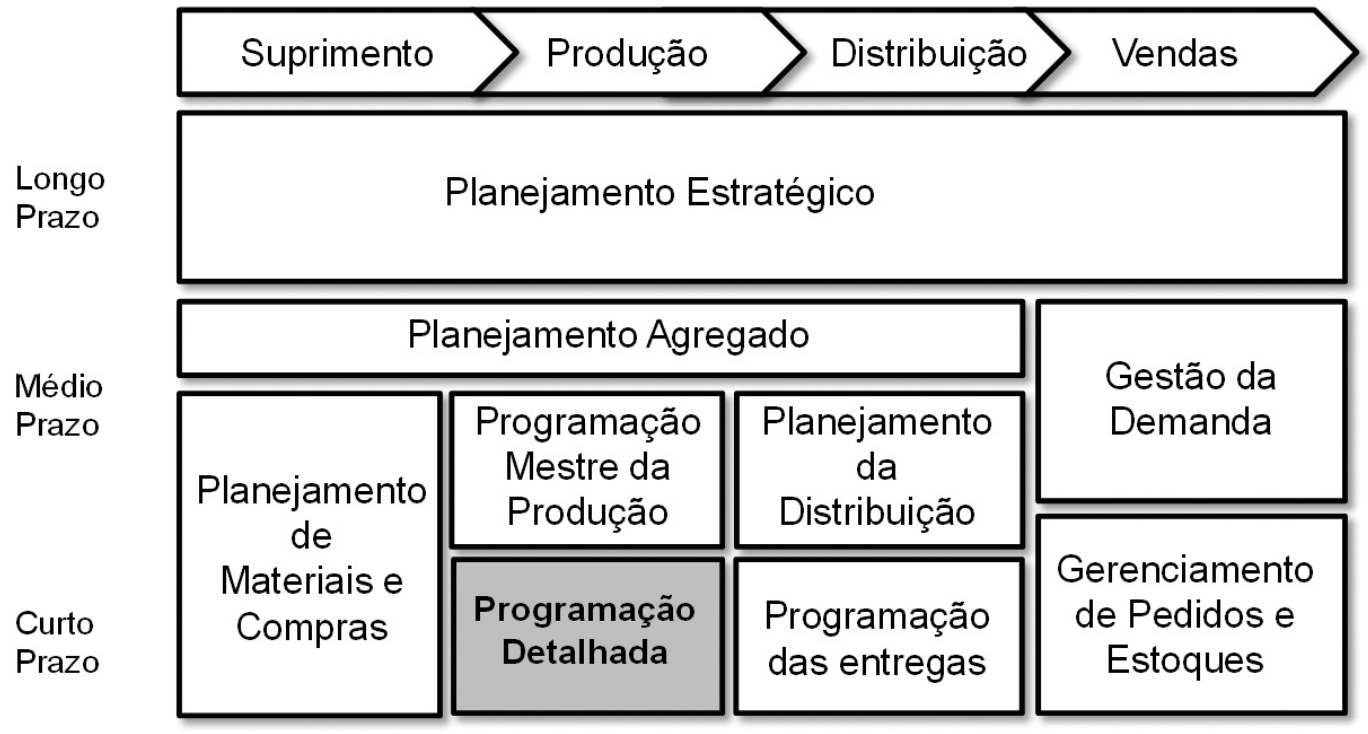

Figura 2. Módulos de um sistema APS. Fonte: adaptado de Meyr et al. (2005). 
lvert \& Jonsson (2010) estudaram os benefícios potenciais do uso do APS no processo de S\&OP (Sales and Operations Planning), elencando os benefícios em três classes: suporte à decisão, planejamento eficiente e construção de aprendizado.

Embora os sistemas APS tenham evoluído de tal forma que podem ser utilizados de forma autônoma, visto que muitos deles possuem a opção de cadastrar pedidos, roteiros de produção e recursos, na maioria das vezes eles se integram ao sistema ERP corporativo (Ivert \& Jonsson, 2010).

Deve-se acrescentar o fato de que os sistemas ERPs são essencialmente transacionais e o que o pessoal encarregado da programação nas empresas realmente requer são ferramentas analíticas, que ajudem a avaliar as alternativas de solução e a tomada de decisão em um curto espaço de tempo, foco dos sistemas APS (Faé \& Erhart, 2009).

\subsection{Programação detalhada mediante $M R P$ versus $A P S / P F$}

No que se refere à programação detalhada da produção, merece destaque o fato de que o MRP gera efetivamente um conjunto de ordens de produção (fabricação e montagem). No entanto, Hopp \& Spearman (2008) destacam algumas críticas recorrentes ao MRP:

- Programação infinita: 0 MRP considera que os lead times das ordens são constantes (atributos dos itens) e não dependem do status do chão de fábrica, o que equivale a dizer que assume capacidade infinita dos recursos;

- Lead times superestimados: É comum que lead times fixos sejam sobrestimados, de maneira que o pedido tenha maiores possibilidades de ser completado a tempo. lsso provoca lançamentos antecipados, filas e tempos de fluxo maiores;

- Nervosismo do sistema: Entende-se o efeito que ocorre quando pequenas variações no MPS produzem grandes mudanças no MRP devido, entre outros fatores, às políticas de lote.

No MRP, o tempo de fila de cada operação está incluso no lead time estimado, enquanto nos sistemas com capacidade finita esse tempo é calculado (é uma saída do sistema). Além disso, o MRP utiliza no planejamento uma grade com janelas de tempo (time buckets) predefinidas, usualmente dias ou semanas. Já no APS/PF o tempo pode ser medido continuamente (horas e minutos), proporcionando que as operações sejam programadas com maior nível de detalhe. Vale dizer que o MRP considera o tempo discreto, enquanto os APS/PF o consideram contínuo.
Critica-se o MRP também pela cultura de formação de lotes, que pode gerar ineficiências para a produção em sistemas complexos, gerando excesso de WIP e filas. Nos sistemas APS/PF os lotes estão associados às ordens e, como o modelo avalia sequências, pode-se considerar tempos de set up dependentes da sequência (matriz de set up).

A diferença central entre os sistemas está na forma como são consideradas as restrições de capacidade. No MRP, a programação é gerada com carga ilimitada (supõe-se que tudo que é planejado pode ser executado dentro dos lead times cadastrados no sistema). As verificações de capacidades incluídas posteriormente não proporcionam um nivelamento automático da carga de trabalho nos centros. Os APS/ $\mathrm{PF}$, por considerarem a capacidade dos centros de maneira finita, não geram esse inconveniente (Hopp \& Spearman, 2008).

Corrêa \& Corrêa (2009) explicam que o MRP é tipicamente um sistema de programação para trás com capacidade infinita, enquanto os APS/PF geralmente são sistemas de programação finita para a frente. Alguns sistemas APS/PF utilizam ambas as técnicas (programação bidirecional). Primeiro fazem a programação para trás, tentando diminuir o nível de WIP e, num segundo passo, utilizam a programação para a frente para eliminar eventuais atrasos na programação.

Em síntese, no âmbito da programação detalhada da produção, o MRP 11 (ou o ERP) não fornece uma programação adequada principalmente devido ao método de carga que pressupõe capacidade infinita dos recursos. Essa limitação, paralelamente ao desenvolvimento da tecnologia de informação, fomentou o desenvolvimento dos APS como alternativa de sistema em programação detalhada da produção (Stadtler, 2005; Hopp \& Spearman, 2008; Hvolby \& Steger-Jensen, 2010).

A Tabela 1 resume algumas diferenças entre as abordagens MRP e APS na Programação Detalhada da Produção (Girotti \& Mesquita, 2011).

\section{Estratégias de ensino}

0 modelo de ensino tradicional, focado principalmente na aula expositiva, enfrenta dificuldades na era da informação devido à grande disponibilidade delas, principalmente via internet.

Segundo Cintra (2012), o tipo de aula conteudista piorou com a aparição do data-show (ou projetor), que permitiu ao professor incluir muito mais conteúdo do que antes, correndo o risco de que seus alunos se "percam" entre tanta informação nova, o que se 
Tabela 1. Diferenças entre as abordagens MRP e APS/PF na programação detalhada.

\begin{tabular}{|l|l|l|}
\hline \multicolumn{1}{|c|}{ Tópico } & \multicolumn{1}{c|}{ MRP } & \multicolumn{1}{c|}{ APS/PF } \\
\hline Foco & Viabilidade no plano de materiais & Otimização da capacidade \\
\hline Dificuldade & Balancear a carga dos centros & Administrar necessidade de materiais \\
\hline Tempos de fila & Estimados, incluído no lead time & Calculado pelo sistema \\
\hline Capacidade & Carga ilimitada & Carga limitada pela capacidade \\
\hline Unidades de tempo & Discreto (time buckets): dias ou semanas & Contínuo: horas e minutos \\
\hline Orientação & Programação para trás com capacidade infinita & $\begin{array}{l}\text { Programação para a frente com capacidade finita (para } \\
\text { alguns, bidirecional) }\end{array}$ \\
\hline Sequenciamento & Indisponível no time bucket & Sim \\
\hline Sistema & Transacional & Apoio às decisões \\
\hline Capacidade de simulação & Baixa & Alta \\
\hline Capacidade de otimização & Baixa & Alta \\
\hline Complexidade & Baixa & Alta \\
\hline
\end{tabular}

Fonte: Girotti \& Mesquita (2011).

traduz em um menor aproveitamento e interesse pelas aulas.

Vários autores discutem a necessidade de mudança desse paradigma no processo de ensino-aprendizagem na engenharia (Belhot, 1997; Silva \& Cecílio, 2007). 0 professor já não teria o papel de detentor do conhecimento, que o transmite para os seus alunos, senão que passaria a ser um "facilitador" da aprendizagem (Belhot, 1997). Para isso, recomenda-se o uso de outras estratégias de ensino.

Segundo Wankat \& Oreovicz (1993), existem diferenças naturais entre todos os seres humanos nas formas de aprendizagem. Estratégias podem ser implantadas de modo a tentar abranger a maior quantidade possivel dessas possibilidades.

De acordo com Cavellucci (2006), as estratégias de aprendizagem são maneiras de lidar com as diferentes formas em que as informações são apresentadas e as situações de aprendizagem são organizadas. Elas visam superar as dificuldades na transmissão do conhecimento e definir quais as preferências de aprendizagem dos alunos serão mais potencializadas. Destaca-se que quanto maior a variedade de estratégias utilizadas, maior será a eficácia das experiências de aprendizagem vivenciadas pelos alunos.

A seguir são apresentadas as duas estratégias de ensino analisadas nesta pesquisa: simulação e estudos de caso.

\subsection{Simulação}

Por simulação entende-se a experimentação com modelos que representam com um determinado grau de precisão uma situação da realidade ou certo processo/fenômeno conhecido. A simulação ajuda a elaborar diferentes cenários para o problema em estudo, permitindo a elaboração de conclusões baseadas em análises do tipo "e se".
As técnicas de simulação podem ser categorizadas basicamente em três grupos: situações simuladas, onde os alunos interpretam papéis; simulações com kits de materiais (tabuleiros, peças, cartas etc.); e, por último, as simulações computacionais (Salas et al., 2009).

A simulação tem sido aplicada em educação de várias formas: jogos de empresa (Sauaia, 2010), simuladores (Mesquita \& Hernandez, 2006), tutoriais (Atolagbe et al., 2001). Jahangirian et al. (2010) apresentam um detalhado levantamento de artigos publicados acerca de simulação nas áreas de produção e negócios, incluindo sua utilização em ensino e treinamentos.

Salas et al. (2009) apresentam um guia para desenvolver e implantar um treinamento baseado em simulação na área de administração que pode ser empregado em outras áreas também.

Chase et al. (2006) enumeram as seguintes vantagens da simulação:

- Criar um modelo geralmente permite entender melhor o sistema real;

- Compressão do tempo: É possível simular meses, anos em minutos;

- Não interrompe as atividades que estão se desenvolvendo no sistema real;

- É mais abrangente que os modelos analíticos;

- Usada como jogo ela proporciona uma experiência para a capacitação;

- Permite analisar condições transitórias, diferente de modelos analíticos;

- Existe no mercado uma variada quantidade de softwares de simulação;

- Permite a elaboração e análise de diferentes cenários. Os mesmos autores enumeram as seguintes desvantagens: 
- 0 desenvolvimento de modelos para sistemas reais complexos pode ser caro e demorado;

- Mesmo com muito investimento na modelagem, não há garantia de que a simulação ofereça respostas conclusivas;

- Os resultados devem ser interpretados estatisticamente;

- A rodada de modelos complexos pode consumir muito tempo no computador;

- Muitos cenários podem ser necessários para uma avaliação mais completa do sistema simulado;

- A modelagem não é um processo padrão, portanto, dois modelos de um mesmo sistema podem gerar resultados (e conclusões) muito diferentes.

\subsection{Estudo de casos didáticos}

0 estudo de caso para ensino pode ser definido de várias formas. Davis \& Wilcock (2012) definem o caso didático como atividades centradas no aluno, com base em elementos que demonstram conceitos teóricos de forma prática.

Para Mintzberg (2004, p. 48):

Um caso é um maço de papéis de cerca de dez a vinte páginas, compostas principalmente de palavras no texto e, muitas vezes, números nos apêndices, às vezes com algumas fotos, que descreve uma situação de negócio geralmente em uma única empresa, com um protagonista em algum tipo de encruzilhada, tendo que tomar uma decisão.

Graham (2010) detalha algumas características dos casos didáticos conforme apresentado na Tabela 2 .

Graham (2010) explica que muitos casos, principalmente os empregados no ensino, não fornecem todos os fatos. 1sso produz um desafio nos alunos, para acrescentarem as suas próprias interpretações.

Conforme Davis \& Wilcock (2012), existem riscos associados a essa estratégia:

- Trabalho em equipe: Geralmente não é fornecido junto com o caso um treinamento nesse tipo de trabalho. Risco de que a participação não seja igual entre os integrantes do grupo;

- Explicação dos requisitos para resolver o caso: Os alunos muitas vezes desejam mais informações sobre o que se espera deles na resolução do caso;
- Nível de aprofundamento da aprendizagem: Em casos onde os alunos devam fazer algum tipo de pesquisa, existe o risco de que tudo o que deve ser pesquisado venha de uma única fonte: internet. Embora ela seja um recurso valioso, geralmente traduz-se em uma aprendizagem não muito profunda;

- Valor da nota: 0 professor tem de decidir o peso do caso didático na nota da disciplina, considerando a carga de trabalho.

\section{Metodologia}

Esta pesquisa pode ser definida, segundo a classificação encontrada em Gil (2008), como uma pesquisa descritiva, onde o objetivo principal é a descrição das características de determinada população ou fenômeno ou, então, o estabelecimento de relações entre variáveis.

0 método de pesquisa empregado é o levantamento por amostragem ou survey com um questionário autoaplicado. Forza (2002) aponta que o survey envolve uma coleta de informações sobre indivíduos ou sobre as unidades sociais (por exemplo, a escola) a que eles pertencem.

Para o levantamento empregou-se um questionário composto de 18 questões. 0 questionário foi dividido em quatro seções, como apresentado na Tabela 3. Empregou-se a validação sugerida por Leedy (2000), apresentada na mesma tabela.

A primeira seção é de identificação do respondente. Decidiu-se que o questionário não seria anônimo, primeiro para assegurar que uma mesma pessoa não respondesse duas vezes, e também, porque foi prometido no e-mail convite que os resultados seriam divulgados entre os participantes. Obviamente, os dados pessoais não são divulgados. A segunda e terceira seções tratam sobre os objetivos da pesquisa. Na seção 2 consulta-se sobre a inclusão e a importância dos temas tratados (MRP e APS). A seção 3 consulta sobre estratégias de ensino (casos e simulação). Para finalizar, foi inserida uma pergunta aberta caso o respondente quisesse realizar algum comentário, sugestões ou críticas.

A ferramenta escolhida para aplicar o questionário foi o software Google Drive. Ele permite a criação de

Tabela 2. Características dos casos didáticos.

\begin{tabular}{|l|l|}
\hline Fonte de informação & Fato ou ficção \\
\hline Objetivos de aprendizagem & Objetivos específicos de aprendizagem (em geral vinculados à teoria) \\
\hline Formatos & Ampla gama de formatos para se adequar a uma situação de ensino \\
\hline Abordagem geral & Extrai lições relevantes para fins de ensino \\
\hline Benefícios individuais & Desenvolve habilidades, de análise de trabalho em grupo, de comunicação, de resolução de problemas etc. \\
\hline Características específicas & Teoria e prática combinadas \\
\hline
\end{tabular}


Tabela 3. Questionário para professores de PCP.

\begin{tabular}{|c|c|c|c|c|c|c|}
\hline \multirow[b]{2}{*}{ Tópico } & \multirow[b]{2}{*}{ Pergunta } & \multirow{2}{*}{$\begin{array}{l}\text { Qual é a razão para } \\
\text { essa questão? }\end{array}$} & \multicolumn{3}{|c|}{ Tipo de questão } & \multirow{2}{*}{$\begin{array}{l}\text { Como você espera } \\
\text { relacionar essa questão } \\
\text { com a pesquisa? }\end{array}$} \\
\hline & & & $\begin{array}{l}\text { Múltipla } \\
\text { escolha }\end{array}$ & Escala & $\begin{array}{l}\text { Questão } \\
\text { aberta }\end{array}$ & \\
\hline \multirow{5}{*}{ Identificação } & 1. Nome completo: & \multirow{5}{*}{$\begin{array}{l}\text { ldentificar e } \\
\text { caracterizar os } \\
\text { respondentes. }\end{array}$} & & & $X$ & \multirow{5}{*}{$\begin{array}{l}\text { Não está diretamente } \\
\text { relacionada. }\end{array}$} \\
\hline & 2. Curso/lnstituição: & & & & $X$ & \\
\hline & 3. Endereço de e-mail: & & & & $X$ & \\
\hline & 4. Titulação: & & $x$ & & & \\
\hline & $\begin{array}{l}\text { 5. Há quantos anos ministra a } \\
\text { disciplina PCP? }\end{array}$ & & $\mathrm{X}$ & & & \\
\hline \multirow{5}{*}{$\begin{array}{l}\text { Tópicos } \\
\text { referentes à } \\
\text { disciplina }\end{array}$} & $\begin{array}{l}\text { 6. Inclui o tópico Material } \\
\text { Requirements Planning - MRP } \\
\text { nas suas aulas? }\end{array}$ & \multirow{4}{*}{$\begin{array}{l}\text { Saber se o } \\
\text { respondente inclui } \\
\text { nas suas aulas os } \\
\text { tópicos MRP e } \\
\text { Programação Finita e } \\
\text { avaliar a importância } \\
\text { que ele lhes dá. }\end{array}$} & $X$ & & & \multirow{4}{*}{$\begin{array}{l}\text { Definir se para os } \\
\text { professores de PCP os } \\
\text { tópicos desta pesquisa são } \\
\text { importantes. }\end{array}$} \\
\hline & $\begin{array}{l}\text { 7. Por favor, avalie a } \\
\text { importância do tópico MRP na } \\
\text { disciplina PCP. }\end{array}$ & & & $x$ & & \\
\hline & $\begin{array}{l}\text { 8. Inclui o tópico Programação } \\
\text { Finita (programação detalhada } \\
\text { considerando capacidade finita } \\
\text { dos recursos) nas suas aulas? }\end{array}$ & & $X$ & & & \\
\hline & $\begin{array}{l}\text { 9. Por favor, avalie a } \\
\text { importância do tópico } \\
\text { Programação Finita na } \\
\text { disciplina. }\end{array}$ & & & $X$ & & \\
\hline & $\begin{array}{l}\text { 10. Por favor, avalie a } \\
\text { importância de diferenciar as } \\
\text { abordagens MRP (capacidade } \\
\text { infinita) e APS (capacidade } \\
\text { finita). }\end{array}$ & Verificar importância. & & $x$ & & $\begin{array}{l}\text { Verificar importância do } \\
\text { problema de pesquisa. }\end{array}$ \\
\hline \multirow{8}{*}{$\begin{array}{l}\text { Estratégias de } \\
\text { ensino }\end{array}$} & $\begin{array}{l}\text { 11. Utiliza técnicas de } \\
\text { simulação na disciplina? }\end{array}$ & \multirow{6}{*}{$\begin{array}{l}\text { Avaliar o grau de } \\
\text { utilização de técnicas } \\
\text { de simulação no } \\
\text { ensino. }\end{array}$} & & $X$ & & \multirow{6}{*}{$\begin{array}{l}\text { Entender se os } \\
\text { respondentes usam } \\
\text { técnicas empregadas nesta } \\
\text { pesquisa e em que tópicos } \\
\text { eles as empregam. }\end{array}$} \\
\hline & $\begin{array}{l}\text { 12. Se utiliza, por favor, indique } \\
\text { que técnicas emprega. }\end{array}$ & & $x$ & & & \\
\hline & 13. Em quais tópicos? & & $x$ & & & \\
\hline & $\begin{array}{l}\text { 14. Emprega estudos de caso } \\
\text { na disciplina? }\end{array}$ & & & $x$ & & \\
\hline & $\begin{array}{l}\text { 15. Se emprega, por favor, } \\
\text { indique que tipo de casos usa. }\end{array}$ & & $x$ & & & \\
\hline & 16. Em quais tópicos? & & $\mathrm{X}$ & & & \\
\hline & $\begin{array}{l}\text { 17. Avalie a seguinte frase: "0 } \\
\text { uso de estudos de caso com } \\
\text { uso de software (aplicativos) } \\
\text { contribui para a melhoria do } \\
\text { aprendizado em PCP". }\end{array}$ & Verificar importância. & & $x$ & & $\begin{array}{l}\text { Saber a importância que } \\
\text { os respondentes dão à } \\
\text { solução proposta para o } \\
\text { problema desta pesquisa. }\end{array}$ \\
\hline & $\begin{array}{l}\text { 18. Se emprega estudos de } \\
\text { caso com uso de software } \\
\text { (aplicativos), favor relatar } \\
\text { brevemente sua experiência. }\end{array}$ & Conhecimento. & & & $x$ & $\begin{array}{l}\text { Conhecer a experiência } \\
\text { de outros professores na } \\
\text { questão de pesquisa. }\end{array}$ \\
\hline Comentários & $\begin{array}{l}\text { Sinta-se à vontade para realizar } \\
\text { comentários, sugestões e } \\
\text { críticas. }\end{array}$ & $\begin{array}{l}\text { Outras opiniões dos } \\
\text { respondentes não } \\
\text { contempladas nas } \\
\text { perguntas anteriores. }\end{array}$ & & & $\mathrm{X}$ & $\begin{array}{l}\text { Compilar outras } \\
\text { informações que possam } \\
\text { ajudar na resolução do } \\
\text { problema de pesquisa. }\end{array}$ \\
\hline
\end{tabular}

formulários na forma de questionários e o seu envio na forma de um hiperlink. 0 software também compila as respostas, apresentando os resultados em formato de planilha. Permite também exportar os resultados em diferentes formatos (planilha, texto plano etc.).

Assim, foi realizado o seguinte procedimento para elaboração do cadastro dos respondentes: utilizando-se a lista de cursos de graduação em Engenharia de Produção publicados no site da ABEPRO (Associação Brasileira de Engenharia de Produção), foram procurados e-mails de contato para envio.
Consideraram-se válidos endereços de coordenadores do curso, secretárias e, obviamente, de professores de disciplinas de Planejamento e Controle da Produção. Para completar essa tarefa, o autor procurou em cada um dos sites publicados de cada universidade algum endereço de e-mail de contato.

Como resultado desse levantamento, entre os 486 cursos listados e acessados foram apontados 178 endereços para contato. Para todos eles foi enviado um e-mail convite. Nele foram inseridas duas alternativas de acesso ao questionário: um hiperlink fornecido 
pelo Google Drive, e outro hiperlink que levava ao site da universidade dos autores deste artigo, onde também podia ser acessado o link da Google (isso foi feito para dar mais credibilidade ao e-mail convite). 0 total de respostas coletadas foi de 53 , alcançando uma taxa de retorno de quase $30 \%$. No recebimento de cada resposta, foi enviado ao participante um e-mail de agradecimento.

Dado que a amostragem pode ser considerada como não probabilística, os dados obtidos serão tratados qualitativamente. Na seção seguinte são apresentados e discutidos esses resultados.

\section{Discussão dos resultados}

Começando a discussão dos resultados do questionário, as perguntas 1 a 3 (nome completo, curso e endereço de e-main), por serem sobre informação confidencial, não serão comentadas.

\section{Titulação}

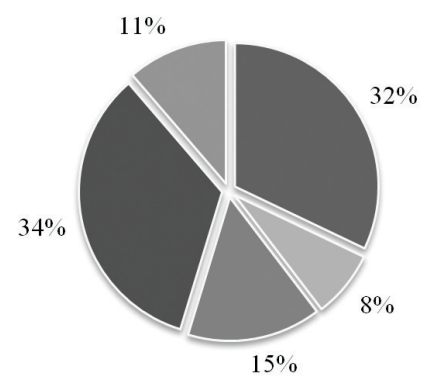

- Professor Titular

Professor Associado

- Professor Doutor

- Professor Assistente

- Outro

Figura 3. Titulação dos respondentes.

Tabela 4. Quantidade de anos ensinando a disciplina PCP.

\begin{tabular}{lcc}
\hline 5. Há quantos anos ministra a disciplina PCP? & Total & $\%$ \\
\hline Menos de 2 anos & 16 & 30 \\
Entre 2 e 5 anos & 18 & 34 \\
Entre 5 e 10 anos & 11 & 21 \\
Mais de 10 anos & 8 & 15 \\
Total geral & 53 & 100 \\
\hline
\end{tabular}

A Figura 3 classifica os respondentes pela titulação. A maior porcentagem encontra-se entre professores titulares e assistentes.

Completando, a Tabela 4 indica há quantos anos o professor ministra a disciplina. Não existe uma categoria dominante, no entanto pode-se dizer que a maioria (64\%) ministra há menos de cinco anos.

As perguntas 6 a 10 avaliam se para os respondentes os tópicos MRP e Programação Finita são temas importantes para a disciplina. A pergunta 6 (Inclui o tópico MRP na sua disciplina?) teve resposta afirmativa em 100\% dos casos. Consultados, na pergunta 7 , sobre a importância desse tópico na disciplina, em uma escala de 1 - Não importante, até 5 - Muito importante, 91\% consideraram o MRP um tema importante ou muito importante (Figura 4).

Perguntas similares foram feitas para Programação Finita. Na pergunta 8 questionou-se se esse tópico era abordado nas aulas: $81 \%$ dos professores (43 respostas) declararam que sim. Já na pergunta 9 , $72 \%$ responderam que o tema é importante ou muito importante (Figura 5). Observa-se uma diminuição em comparação com o MRP, um tema mais conhecido da disciplina. Interessante é que das seis respostas associadas aos menores valores da escala, só três correspondem aos mesmos professores que não incluem o tópico Programação Finita. Portanto, devem existir outras razões para a não inclusão do tema, por exemplo, por falta de tempo.

Finalmente, na pergunta 10 foi questionada a importância do problema de pesquisa deste trabalho. Como mostra a Figura 6, uma alta percentagem (78\%) dos professores consideraram importante ou muito importante diferenciar as abordagens MRP e APS/PF.

Pode-se concluir que a questão de pesquisa é considerada importante por uma parcela considerável dos respondentes.

A terceira seção do questionário trata das estratégias de ensino. Começando pela estratégia simulação, os respondentes foram consultados sobre se utilizavam a estratégia e, no caso de resposta

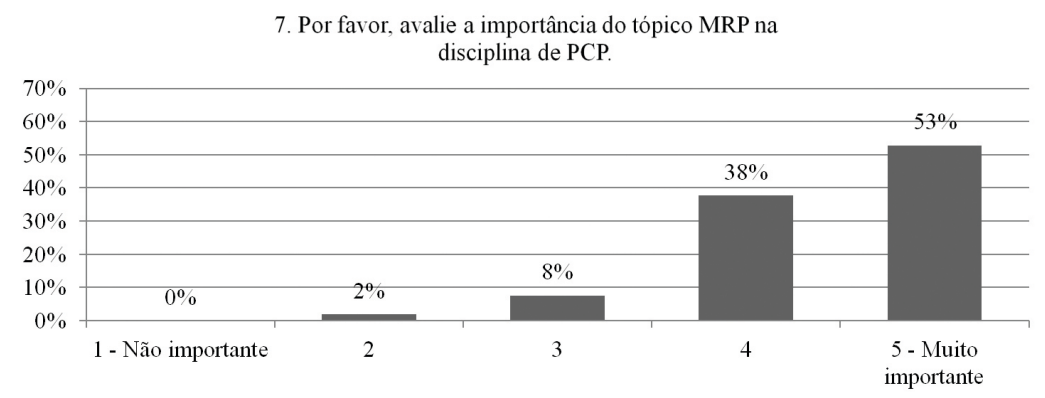

Figura 4. Importância do MRP na disciplina. 
afirmativa, quais técnicas empregavam e em quais temas. Os resultados podem ser visualizados nas Figuras 7, 8 e 9. Nas últimas duas perguntas, os respondentes podiam escolher mais de uma opção, por isso o total de respostas é maior que o número de respondentes.

Pode-se concluir que a simulação computacional é uma técnica razoavelmente empregada (64\% somados os valores 4 e 5 da escala). Pelo observado na Figura 8, a grande maioria utiliza simulação em planilhas, seguida de situações simuladas. Geralmente esses tipos de simulações são as mais fáceis de implantar, devido à disponibilidade de recursos. Dentre os tópicos tradicionais da disciplina, a Figura 9 expõe que os mais ensinados usando simulação são Previsão de Demanda, MRP, Controle de Estoques e MPS. Os três temas inclusos na opção Outro foram Teoria das Restrições, Planejamento de Capacidade e PERT/CPM.

As próximas três questões (14 a 16), com respostas apresentadas nas Figuras 10, 11 e 12, são equivalentes às anteriores, mas voltadas ao uso de estudos de caso.

0 uso de casos também obteve $64 \%$ entre os dois valores mais altos da escala, o que é considerado um valor alto.

Na Figura 11 tem-se um empate no uso de casos didáticos (entendidos aqui como casos com dados fictícios) e casos reais encontrados na literatura. 0 valor descrito em Outro foi Indústria, presumivelmente seja um caso real empregado pelo respondente.

Observando-se a Figura 12 pode-se ver que no tema MRP o caso é muito empregado (37 de 53

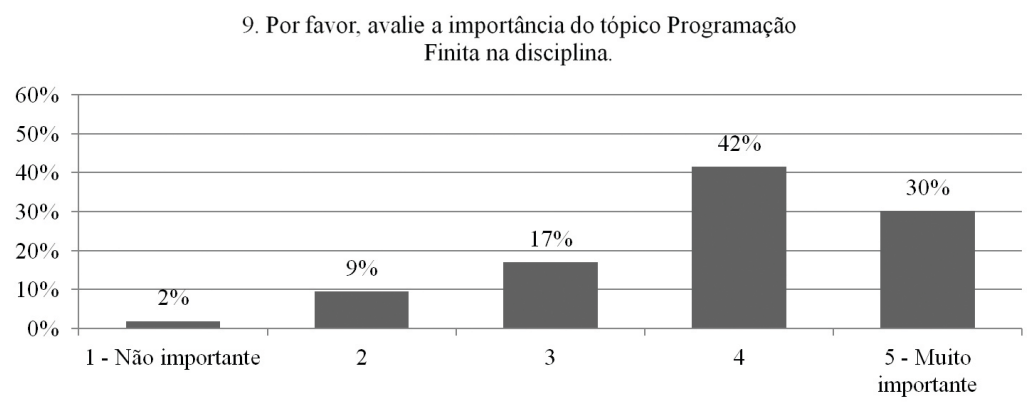

Figura 5. Importância da PDP na disciplina.

10. Por favor, avalie a importância de diferenciar as abordagens MRP (capacidade infinita) e APS (capacidade finita).

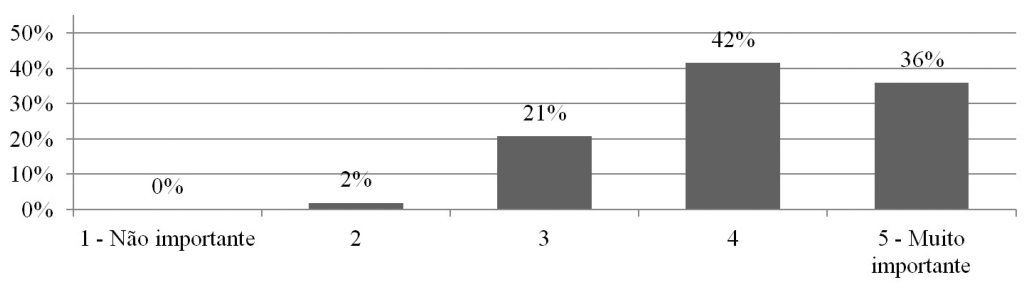

Figura 6. Importância de diferenciar as abordagens MRP e APS/PF.

11. Utiliza técnicas de simulação na disciplina?

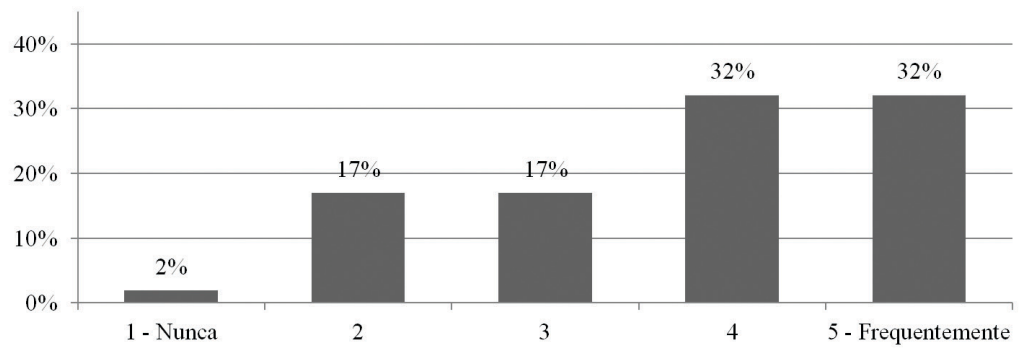

Figura 7. Uso de simulação na disciplina PCP. 
12. Se utiliza, por favor, indique que técnicas emprega.

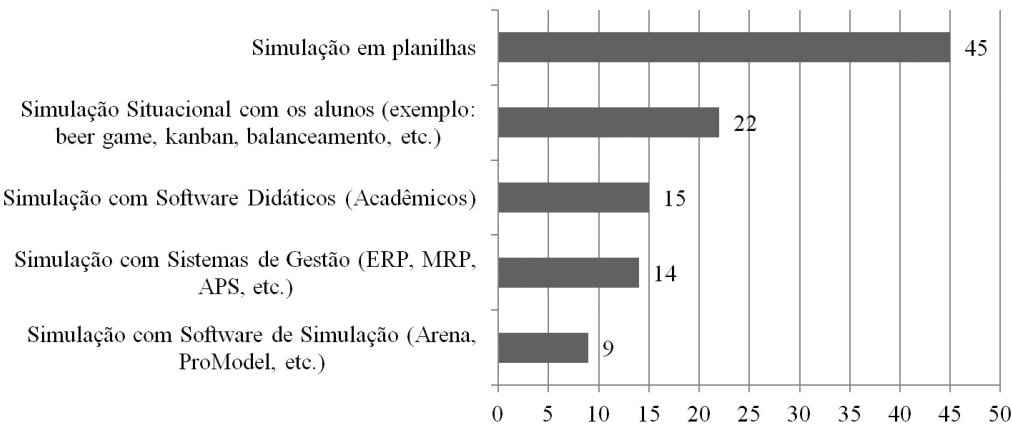

Figura 8. Técnicas de simulação empregadas em PCP.

13. Em quais tópicos emprega simulação?

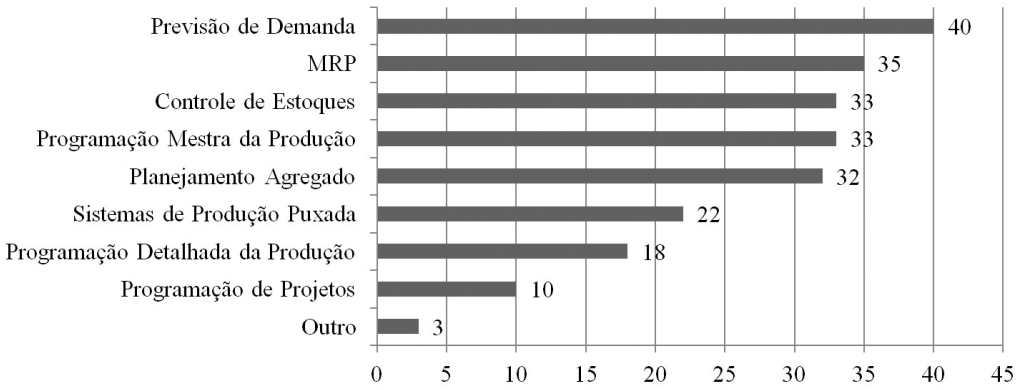

Figura 9. Uso de simulação em PCP por temas.

14. Emprega estudos de caso na disciplina?

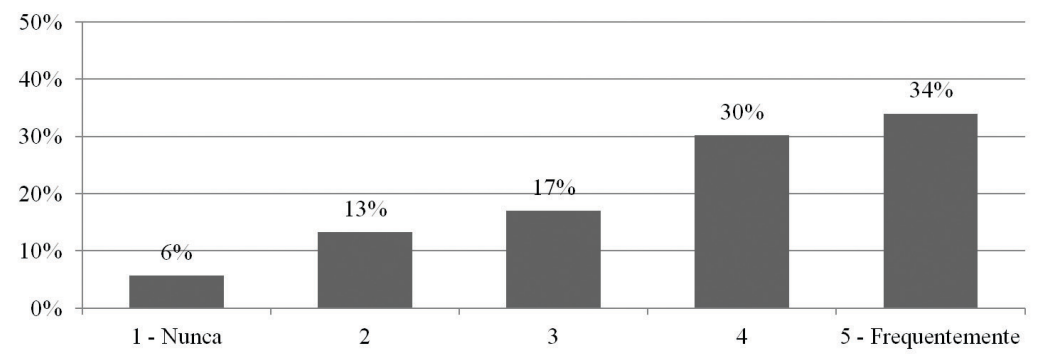

Figura 10. Uso de casos na disciplina PCP.

15. Se emprega, por favor, indique que tipo de casos usa.

Casos didáticos - elaboração própria

Casos didáticos - elaborados por terceiros

Estudos de Casos reais publicados na literatura

Estudos de Casos elaborados pelos alunos durante a disciplina

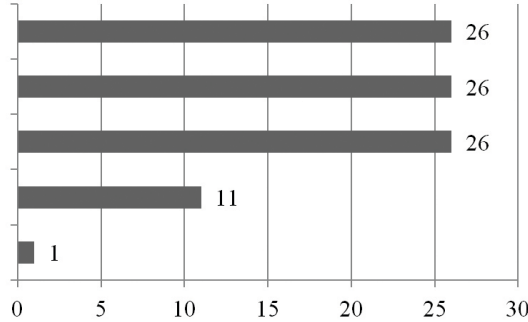

Figura 11. Estudos de caso empregados em PCP. 


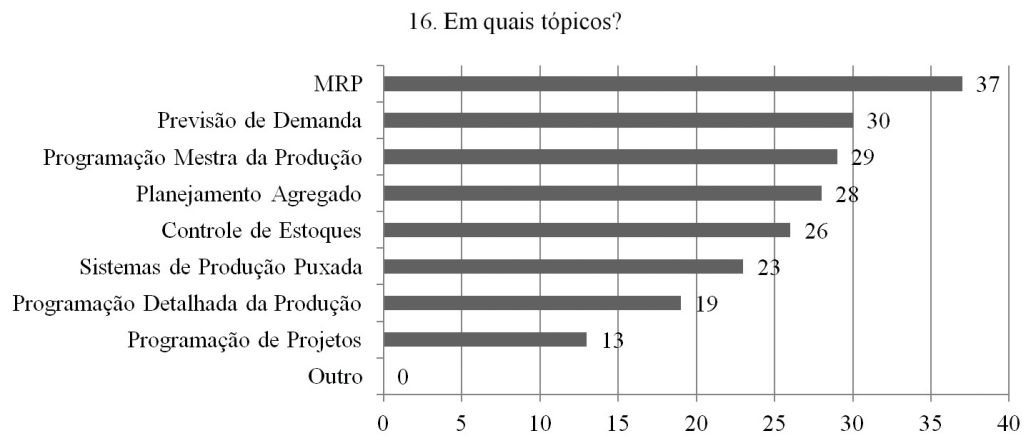

Figura 12. Uso de casos em PCP por temas.

17. Avalie a seguinte frase: "O uso de estudos de caso com uso de software (aplicativos) contribuem para melhoria do aprendizado em PCP".

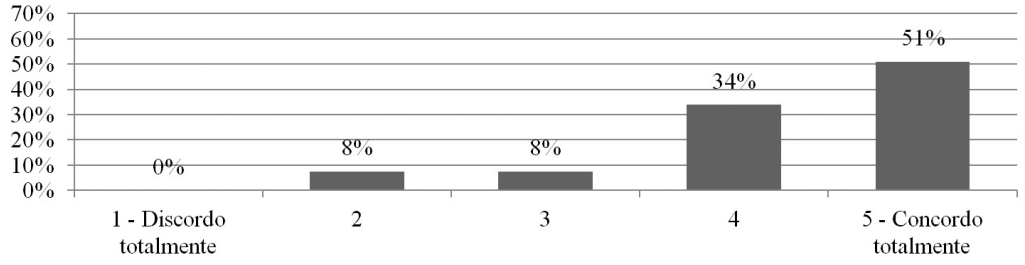

Figura 13. Concordância com o uso de casos em PCP.

Tabela 5. Resposta sobre experiência de uso de casos e softwares.

\begin{tabular}{lc}
\multicolumn{1}{c}{ Conceito } & $\begin{array}{c}\text { Quantidade } \\
\text { de respostas }\end{array}$ \\
\hline Uso de planilhas de cálculo & 3 \\
Uso de softwares acadêmicos & 3 \\
Caso de dimensões reduzidas e simulação & 1 \\
Caso real referente a atividades de consultoria & 1 \\
Caso próprio & 1 \\
Simulação situacional (uso de Lego) e módulos & 1 \\
de SAP/R3 & 1 \\
Uso de software comercial & 11 \\
Total & \\
\hline
\end{tabular}

Tabela 6. Comentários, críticas e sugestões dos professores.

\begin{tabular}{lc}
\hline \multicolumn{1}{c}{ Conceito } & $\begin{array}{c}\text { Quantidade } \\
\text { de respostas }\end{array}$ \\
\hline Dificuldade de acesso a software & 3 \\
Pouca valorização por parte da Coordenação & 2 \\
Poucos exemplos de casos em livros & 2 \\
$\begin{array}{l}\text { Disponibilidade limitada de horas-aula para discutir } \\
\text { casos }\end{array}$ & 1 \\
$\begin{array}{l}\text { Uso de simulação para modelagem } \\
\text { Preferência pelo uso de planilhas de cálculo }\end{array}$ & 1 \\
Professor não achou software que atenda suas & 1 \\
expectativas & 1 \\
Total & 11 \\
\hline
\end{tabular}

respondentes o usam). Já em Programação Detalhada o uso é menor, só 19 respondentes declararam empregá-lo. Espera-se que a publicação desses resultados provoque uma reflexão sobre o emprego de casos em PDP.

Na penúltima pergunta desta seção (Figura 13), $85 \%$ dos professores concordaram que o uso de estudos de casos apoiados por aplicativos contribui para o aprendizado em PCP. Portanto, acredita-se que a estratégia de implantar um caso didático pode ser bem recebida e facilmente replicada.

Concluindo o questionário, foram inclusas duas perguntas abertas. A primeira, número 18 do survey, solicitava aos professores que empregavam casos e simulação que comentassem brevemente a sua experiência. Ao total foram 11 respostas consideradas válidas. Um resumo por conceito chave pode ser observado na Tabela 5.

Para finalizar, a última questão também é aberta e é nela que os respondentes podem incluir comentários, críticas e sugestões. Na Tabela 6 apresenta-se um resumo das respostas.

\section{Conclusões}

Nesta pesquisa procurou-se determinar a importância de abordar em sala de aula a diferenciação entre as abordagens MRP e APS/PF para a programação 
detalhada da produção e verificar o uso de duas estratégias de ensino: simulação e estudos de caso.

As respostas recolhidas indicam que o tópico MRP, sendo um tema "clássico" da disciplina, é amplamente abordado (100\% dos respondentes o abordam e 91\% o consideram importante). Já os valores para o APS/ PF são menores (81\% e 72\%, respectivamente). Assim mesmo, 78\% dos respondentes escolheram os valores mais altos da escala ao serem consultados sobre a importância de diferenciar as abordagens, dando relevância ao problema de pesquisa.

Sobre as estratégias de ensino, o uso da simulação obteve um valor razoavelmente alto (64\% entre os dois valores mais altos da escala). No entanto, seu uso em programação detalhada da produção não foi elevado (18 de 53 declararam usá-lo). Os resultados para a estratégia estudo de caso foram muito similares, obtendo também 64\%, sendo que 19 professores afirmaram usar casos para programação detalhada.

Com esses resultados pode-se inferir que casos didáticos e simulação são estratégias de ensino a serem consideradas na hora de apresentar os temas aos alunos. Descrições de experiências exitosas envolvendo essas estratégias poderiam aumentar o número de adeptos entre os professores.

Como desdobramento da presente pesquisa, encontra-se em desenvolvimento um caso didático focado em Programação Detalhada da Produção.

\section{Referências}

Arnold, J. R. T. (2011). Introduction to Materials Management (7 ed.). Upper Saddle River: Prentice Hall.

Atolagbe, T., Hlupic, V., Taylor, \& S. J. E. (2001). GeNisa: a web-based interactive learning environment for teaching simulation modeling. In Proceedings of The 2001 Winter Simulation Conference, Arlington. http://dx.doi. org/10.1109/WSC.2001.977492

Belhot, R. V. (1997). Reflexões e propostas sobre o "ensinar Engenharia” para o século XXI (Tese de livre docência). Escola de Engenharia de São Carlos, Universidade de São Paulo, São Carlos.

Cavellucci, L. C. B. (2006). Estilos de Aprendizagem: em busca das diferenças individuais. Recuperado em 15 de maio de 2013, de http://www.faculdadebarretos.edu.br/v3/ faculdade/ imagens/nucleo-apoio-docente/ESTILOS\%20 DE\%20 APRENDIZAGEM\%202.pdf.

Chase, R. B., Jacobs, F. R., \& Aquilano, N. J. (2006). Administração da produção para a vantagem competitiva (10 ed.). Porto Alegre: Bookman.

Cintra, C.A. (2012). Reinventando a aula expositiva. São Carlos: Editora Compacta.

Corrêa, H. L., \& Corrêa, C. A. (2009). Administração de produção e operações. Manufatura e serviços: uma abordagem estratégica (2. ed.). São Paulo: Atlas.

Davis, M. M., Aquilano, N. J., \& Chase, R. B. (2001). Fundamentos de Dirección de Operaciones (3. ed.). McGraw-Hill, Interamericana de España, S.A.U.
Davis, C., \& Wilcock, E. (2012). Teaching Materials using Case Studies. UK Centre for Materials Education. Recuperado em 15 de maio de 2013, de http://www.materials.ac.uk/ guides/casestudies.asp.

Dumond, E. J. (2005). Understanding and using the capabilities of finite scheduling. Industrial Management \& Data Systems, 105(4), 506-526. http://dx.doi. org/10.1108/02635570510592398

Faé, C., \& Erhart, A. (2009). Desafios e tendências na aplicação de sistemas APS. Mundo Logística, 10, 52-60.

Forza, C. (2002). Survey research in operations management: a process-based perspective. International Journal of Operation \& Production Management, 22(2), 152-194. http://dx.doi.org/10.1108/01443570210414310

Gil, A. C. (2008). Como elaborar Projetos de Pesquisa. (4 ed.). São Paulo: Atlas.

Girotti, L. J., \& Mesquita, M. A. (2011). Uma análise comparativa das abordagens MRP e APS para Programação detalhada da produção. In Anais do XIV Simpósio de Administração da Produção, Logística e Operações Internacionais, São Paulo.

Graham, A. (2010). Como escrever e usar estudos de caso para ensino e aprendizagem no setor público. Brasília: ENAP.

Harrison, P. K., \& Petty, D. J. (2002). Systems for Planning and Control in Manufacturing. Oxford: Elsevier Science.

Herrmann, J. W. (2006). Handbook of Production Scheduling. New York: Springer.

Hopp, W. J., \& Spearman, M. L. (2008). Factory Physics: foundations of manufacturing management (3 ed.). New York: McGraw-Hill/lrwin.

Hvolby, H., \& Steger-Jensen, K. (2010). Technical and industrial issues of Advanced Planning and Scheduling (APS) systems. Computers in Industry, 61, 845-851. http://dx.doi.org/10.1016/j.compind.2010.07.009

lvert, L; \& Jonsson, P. (2010). The potential benefits of advanced planning and scheduling systems in sales and operations planning. Industrial Management \& Data Systems, 110, 659-681. http://dx.doi. org/10.1108/02635571011044713

Jacobs, F. R., \& Weston Junior, F. C. (2007). Enterprise resource planning (ERP) - A brief history. Journal of Operation Management, 25, 357-363. http://dx.doi. org/10.1016/j.jom.2006.11.005

Jahangirian, M., Eldabi, T., Naseer, A., Stergioulas, L. K., \& Young, T. (2010). Simulation in manufacturing and business: A review. European Journal of Operational Research, 203, 1-13. http://dx.doi.org/10.1016/j. ejor.2009.06.004

Leedy, P. D. (2000). Practical Research: planning and design (7 ed.). New Jersey: Prentice-Hall. PMid:10920127.

Lustosa, L., Mesquita, M. A., Quelhas, 0., \& Oliveira, R.J. (2008). Planejamento e Controle da Produção. Rio de Janeiro: Elsevier: Campus.

Mesquita, M. A., \& Hernandez, A. E. (2006). Discrete-event simulation of queues with spreadsheets: a teaching case. In Proceedings of the 2006 Winter Simulation Conference, Monterrey. http://dx.doi.org/10.1109/ WSC.2006.323053

Meyr, H., Wagner, M., \& Rohde, J. (2005). Structure of Advanced Planning Systems. In H. Stadtler, \& C. Kilger, Supply Chain Management and Advanced Planning: Concepts, Models, Software and Case Studies (3 ed.). 
Heidelberg: Springer. PMid:16267227. http://dx.doi. org/10.1007/3-540-24814-5_6

Mintzberg, H. (2004). Managers, Not MBAs: A Hard Look at the Soft Practice of Managing and Management Development. San Francisco: Berrett-Koehler Publishers.

Narasimhan, S. L., Mcleavey D. W., \& Billington, P. J. (1995). Production Planning and Inventory Control. Englewood Cliffs: Prentice Hall.

Orlicky, J. (1975). Material Requirements Planning: the new way of life in production and inventory management. New York: McGraw-Hill.

Ovacik, 1. M. (2011). Advanced Planning and Scheduling Systems: The Quest to Leverage ERP for Better Planning. In K. G. Kempf, P. Keskinocak, \& R. Uzsoy, Planning Production and Inventories in the Extended Enterprise. A State of the Art Handbook (Vol. 1). New York: Springer.

Plenert, G., \& Kirchmier, B. (2000). Finite Capacity Scheduling: management, selection and implementation. New York: John Wiley \& Sons. PMid:10915378.

Salas, E., Wildman, J. L., \& Piccolo, R. F. (2009). Using Simulation-Based Training to Enhance Management Education. Academy of Management Learning \& Education, 8(4), 559-573. http://dx.doi.org/10.5465/ AMLE.2009.47785474

Sauaia, A. C. A. (2010). Laboratório de gestão: simulador organizacional, jogo de empresas e pesquisa aplicada (2. ed.). Barueri: Manole.

Silva, L. P., \& Cecílio, S. (2007). A mudança no modelo de ensino e de formação na Engenharia. Educação em
Revista, 45, 61-80. http://dx.doi.org/10.1590/S010246982007000100004

Slotnick, S. A. (2011). Order acceptance and scheduling: A taxonomy and review. European Journal of Operational Research, 212, 1-11. http://dx.doi.org/10.1016/j. ejor.2010.09.042

Stadtler, H. (2005). Supply Chain Management and Advanced Planning - basics, overview and challenges. European Journal of Operational Research, 163(3), 575-588. http://dx.doi.org/10.1016/j.ejor.2004.03.001

Stevenson, M., Hendry, L. C., \& Kingsman, B. G. (2005). A review of production planning and control: the applicability of key concepts to the make-to-order industry. International Journal of Production Research, 43(5), 869-898. http:// dx.doi.org/10.1080/0020754042000298520

Wankat, P. C., \& Oreovicz, F. S. (1993). Teaching Engineering. New York: McGraw-Hill.

Wight, 0. W. (1984). Manufacturing Resources Planning. MRPII: Unlocking America's Productivity Potential (Rev. ed.). Essex Junction: 0. Wight.

\section{Agradecimentos}

0 presente trabalho faz parte de um projeto de pesquisa financiado mediante bolsa do CNPq, Conselho Nacional de Desenvolvimento Científico e Tecnológico - Brasil.

\title{
Production Planning and Control: a survey of teachers in Production Engineering
}

\begin{abstract}
The creation of value depends on the competitive priorities (cost, quality, flexibility, service and delivery) that will support a company's strategy. The role of Production Planning and Control Systems is to provide information and models for the efficient management of manufacturing resources. This research addresses the teaching of MRP and APS/FCS (finite capacity scheduling) in PPC undergraduate courses. The objectives include reviewing the teaching practices in production scheduling, focusing on simulation and case study strategies and determining whether teachers differentiate between the two main approaches. A survey was conducted for this purpose. The respondents were teachers who lecture in Brazilian Production Engineering courses. The results indicate that MRP is covered by $100 \%$ of the teachers consulted. For APS/FCS, that percentage is $81 \%$. In addition, $78 \%$ consider relevant to differentiate between MRP and APS/FCS, and 85\% agree that the case studies with software promote the learning process.
\end{abstract}

\section{Keywords}

Teaching. Production planning and control. Survey. 